\title{
Probable disseminated Mycobacterium abscessus subspecies bolletii infection in a patient with idiopathic CD4+ T lymphocytopenia: a case report
}

\author{
Claudia Colomba ${ }^{1,2}$, Raffaella Rubino ${ }^{1,2^{*}}$, Paola Di Carlo ${ }^{1,2}$, Caterina Mammina ${ }^{1,2}$, Celestino Bonura ${ }^{1}$, \\ Lucia Siracusa ${ }^{1,2}$, Lucina Titone ${ }^{1,2}$ and Laura Saporito ${ }^{1,2}$
}

\begin{abstract}
Introduction: Rapidly growing mycobacteria are opportunistic pathogens in patients with underlying risk factors. Mycobacterium abscessus subsp. bolletii is a newly recognized member of rapidly growing mycobacteria, isolated from respiratory tract and cutaneous infections.

Case presentation: We describe a case of chronic disseminated infection caused by M. abscessus subsp. bolletii in a 38-year-old Sri Lankan man with idiopathic CD4+ T lymphocytopenia. Idiopathic CD4+ T lymphocytopenia is a rare cause of immunodysfunction that, similar to human immunodeficiency virus infection, causes a depletion of CD4+ T lymphocytes. M. abscessus subsp. bolletii infection was diagnosed by culture isolation from two sputum samples.

Conclusions: To the best of our knowledge this is the first report of $M$. abscessus subsp. bolletii disseminated infection in a patient affected by idiopathic CD4+ T lymphocytopenia. In contrast to previous reports, the isolate of M. abscessus subsp. bolletii presented intermediate resistance to clarithromycin and was susceptible to cefoxitin and imipenem.
\end{abstract}

Keywords: CD4+ T lymphocytopenia, Clarithromycin, Disseminated infection, Mycobacterium abscessus subsp. bolletii rapidly growing mycobacteria

\section{Introduction}

Rapidly growing mycobacteria (RGM) are opportunistic pathogens in patients with underlying risk factors. These organisms are ubiquitous in soil and water and can cause skin, soft tissue, pulmonary, and central nervous system (CNS) infections and disseminated disease [1]. Mycobacterium abscessus subsp. bolletii is a newly recognized member of the RGM family, isolated from respiratory tract and cutaneous infections [2-7].

Idiopathic $\mathrm{CD} 4+\mathrm{T}$ lymphocytopenia (ICL) is a rare syndrome, defined in 1992 as: (1) absolute CD4+ $\mathrm{T}$ lymphocyte level $<300$ cells $/ \mu \mathrm{L}$ or $<20 \%$ of total lymphocytes at a minimum of two separate time points at

\footnotetext{
* Correspondence: raffaellarubino@libero.it

'Dipartimento di Scienze per la Promozione della Salute, Università di Palermo, Via del Vespro 129, Palermo 90127, Italy

${ }^{2}$ Dipartimento di Scienze per la Promozione della Salute, Sezione di Malattie Infettive, Università di Palermo, Via del Vespro 129, Palermo 90127, Italy
}

least six weeks apart; (2) no serological evidence of human immunodeficiency virus (HIV) infection; (3) the absence of any defined immunodeficiency or therapy associated with depressed levels of CD4+ T cells $[8,9]$. The epidemiologic data do not suggest that the condition is caused by a transmissible agent and the exact incidence of the disease is variable according to different studies. Unlike HIV infection, the decrease in the CD4+ cell counts of patients with ICL is often slow or even absent over time, the number of CD8+ T cells is normal, and the immunoglobulin levels are either normal or slightly low. The pathogenesis of ICL may be multi-factorial. The diminished availability of stem cell precursors, the increased $\mathrm{T}$ cell apoptosis, the defective production of tumor necrosis factor (TNF) $\alpha$ and interferon (IFN) $\gamma$, the CD4+ $\mathrm{T}$ cell antibody production and impaired early biochemical events of the CD3-T cell receptor 
(TCR) pathway may be involved in the development of CD4 $+\mathrm{T}$ cell depletion in patients with ICL. ICL is typically diagnosed in middle age, usually after an opportunistic infection [9]. CD4 + T cells have a key role in the immunological response against mycobacterial infections and other parasitic and viral infections. Cryptococcal and nontuberculous mycobacterial infections are the major presenting opportunistic infections [10]. We describe a case of chronic disseminated infection caused by $M$. abscessus subsp. bolletii in a patient with ICL.

\section{Case presentation}

Our patient was a 38-year-old previous healthy Sri Lankan man who had been living in Italy for two years. At admission only a history of allergic rhinitis was reported and corticosteroids intake was denied. He was diagnosed with a right pleural empyema and tubercular spondylitis. Ziehl-Neelsen staining of his pleural exudate yielded acid-fast bacilli and a biopsy of the pleura showed mycobacterial histopathological features.

Our patient was treated with an anti-tubercular treatment of isoniazid (300mg once a day, orally), ethambutol (400mg three times a day, orally), rifampicin (600mg once a day, orally) and pyrazinamide (500mg three times a day, orally) for one year.

After 15 months, our patient developed seizures and was admitted to an emergency room. Magnetic resonance imaging (MRI) scan of the brain revealed a nodular lesion in the right frontal lobe surrounded by perilesional edema. A thoracic computed tomography (CT) scan showed parenchymal consolidation in both lungs, predominantly in the right side (Figure 1). Acid-fast bacilli were detected on a Ziehl-Neelsen-stained sputum smear and in his bronchial aspirate. A polymerase chain reaction (PCR) investigation for Mycobacterium tuberculosis performed on a bronchial aspirate sample yielded a negative result. A culture of bronchial aspirate yielded nontuberculous mycobacteria. Our patient was moved to our Infectious Diseases Department at the University Hospital of Palermo.

On admission, our patient was afebrile. He complained of having had a cough for several weeks, sputum production and weight loss $(5 \mathrm{~kg}$ during the last two months). On chest auscultation, a reduction of vesicular breath sound was heard in the right lung base. On skin examination, painless mobile subcutaneous nodules, recently appeared, were seen on the frontal region, the scrotum and the left thigh. His abdominal, cardiac and neurological examination results were normal. He was on phenobarbital treatment.

Laboratory analyses showed a white blood cell count of $5.14 \times 10^{3}$ cells $/ \mu \mathrm{L}$, a hemoglobin level of $12.7 \mathrm{~g} / \mathrm{dL}$, and a platelet count of $235 \times 10^{3}$ cells $/ \mu \mathrm{L}$. The total lymphocyte count was 580 cells $/ \mu \mathrm{L}$ and the CD4+ T lymphocyte level was 138 cells $/ \mu \mathrm{L}$, corresponding to $23.9 \%$ of total lymphocytes. His serum immunoglobulin level was within normal limits.

The results of an HIV antibody test conducted on admission were negative, and again negative when the test was repeated two months later. Intra-dermal Mantoux testing results and serology tests for human T-lymphotropic virus (HTLV)1 and HTLV2 were also all negative. IgM and IgG anti-Toxoplasma gondii tests were negative, and cytomegalovirus (CMV) IgG were present. Our patient refused a lumbar puncture. Pneumocystis jirovecii was investigated using sputum samples and the results were negative. Therapy with antitubercular drugs (ethambutol $400 \mathrm{mg}$ three times a day, orally, isoniazid $300 \mathrm{mg}$ a day, orally and pyrazinamide $500 \mathrm{mg}$ three times a day, orally) had been maintained because of a history of tubercular pleurisy and spondylodiscitis. We started clarithromycin (500mg twice a day, orally) and rifabutin (450mg once a day, orally). Ziehl-Neelsen staining, culture and PCR tests for M. tuberculosis performed on three different sputum and urine samples all gave negative results. CD4+ $\mathrm{T}$ lymphocyte counts persisted at under 200cells/ $\mu \mathrm{L}$ until discharge one month later.

Ultrasonography of the nodules revealed confined hyper-echoic areas with small hypo-echoic nodules and spotty blood flow signals in the subcutaneous cellular tissue. Testicular ultrasonography revealed diffuse enlargement and heterogeneous echo texture of the right epididymis, a small hypo-echoic nodule in an enlarged right testis, and a minimal hydrocele.

At discharge, the subcutaneous nodules had almost retreated but a chest radiograph revealed a new parenchymal consolidation area in the left lung. Our patient continued the abovementioned treatment until May 2008, when he arbitrarily stopped hospital follow-up and therapy.

Two years after diagnosis, our patient came back to our facility because of a new episode of seizures. Brain MRI scan findings were unmodified and a chest radiograph revealed multiple infiltrates involving both lungs. His CD4+ T lymphocyte count was 169 cells $/ \mu \mathrm{L}$. ZiehlNeelsen staining of a sputum specimen was positive for acid-fast bacilli, while PCR for M. tuberculosis was negative. While waiting for microbiological culture results, we started a treatment that could act against nontuberculous mycobacteria (clarithromycin $500 \mathrm{mg}$ twice a day, rifabutin $300 \mathrm{mg}$ once a day and ciprofloxacin $500 \mathrm{mg}$ twice a day). Cultures on both solid (Löwenstein-Jensen) and liquid (MGIT ${ }^{\text {su }}$ Becton Dickinson, Sparks, MD USA) media yielded the presence of acid-fast bacilli. The culture isolate was identified by analysis of its characteristics on culture (time for growth, colony morphology, pigment production, ability of the isolate to grow at various temperatures on 5\% 


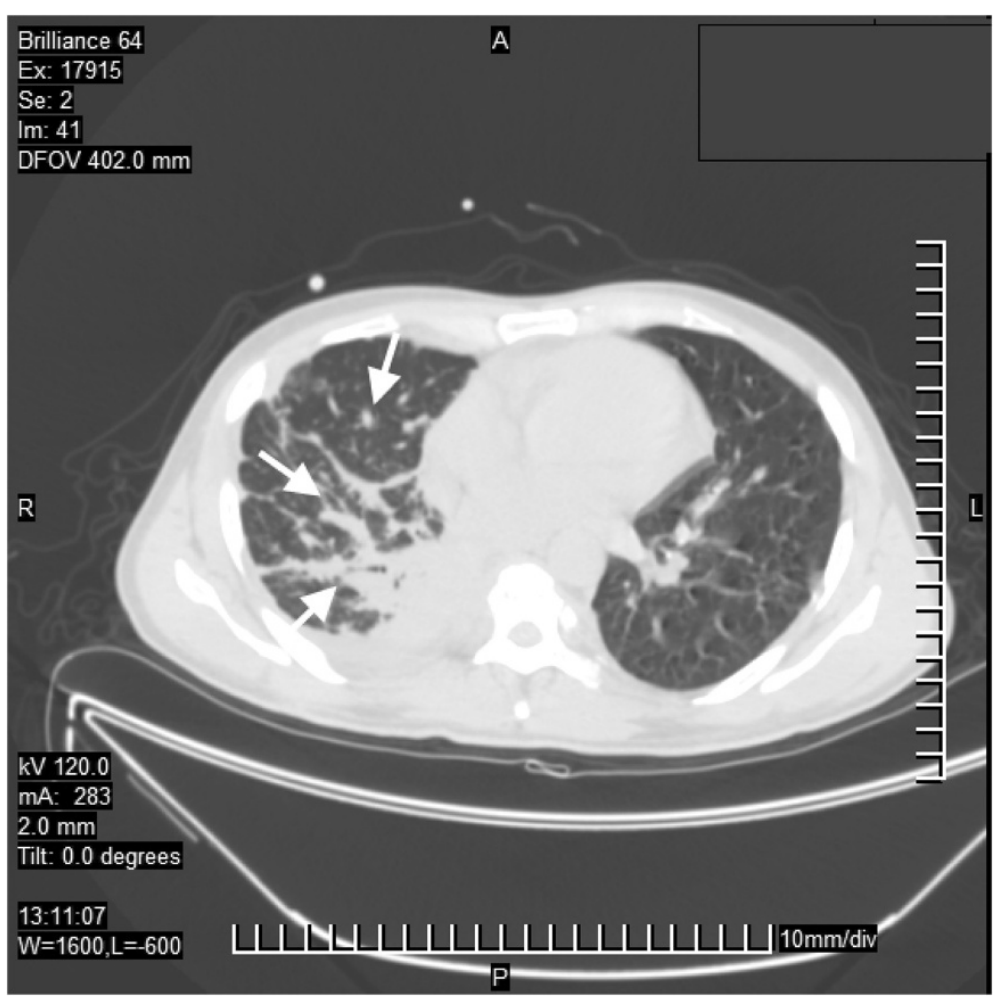

Figure 1 Computed tomography image of lung infection by Mycobacterium abscessus subsp. bolletii. The white arrows show parenchymal consolidation in both lungs, predominantly in the right side, and patchy ground-glass appearance with nodules.

sheep blood agar and Löwenstein-Jensen slants) and by sequence analysis of its ribonucleic acid (RNA) polymerase $\beta$-subunit-encoding gene $(\operatorname{rpoB})$ as $M$. abscessus subsp. bolletii [4]. In particular, after PCR amplification by using the consensus primers described by Adékambi et al. sequences corresponding to $752 \mathrm{bp}$ of the $r$ roB gene were identified by similarity analysis with the sequences in the GenBank database by use of the Basic Local Alignment Search Tool (BLAST) software (http://www.ncbi.nlm.nih.gov/ BLAST). Anti-microbial drug susceptibility tests were performed using the methods described by Adékambi et al. [5]. The results are shown in the Table 1. The results of the anti-microbial susceptibility tests were interpreted according with the criteria proposed by the Clinical and Laboratory Standard Institute (CLSI, formerly NCCLS) and Brown-Elliot et al. $[6,7]$.

Treatment was switched to azithromycin (500mg once a day, orally) and minocycline $(100 \mathrm{mg}$ twice a day, orally). Because of the appearance of inguinal and axillary lymphadenopathy, a biopsy of inguinal lymph nodes was performed and this showed necrotizing granulomatous lymphadenitis. No acid-fast bacilli were seen on ZiehlNeelsen staining. Three months after the first positive culture, M. abscessus subsp. bolletii was again isolated from sputum samples. Our patient is still on therapy and ambulatory follow-up and is in a good clinical condition. We did not perform an MRI because he refused any other diagnostic procedure.

\section{Discussion}

$M$. bolletii is a nontuberculous mycobacteria recently reclassified as a subspecies of $M$. abscessus and renamed M. abscessus subsp. bolletii [2]. It has been described in respiratory tract infections in patients with coexisting broncho-pulmonary diseases and in subcutaneous infections from patients who had undergone mesotherapy $[8,9,11]$.

RGM respiratory disease most often affects patients older than 60 years. Almost all patients younger than 40 years have one of the predisposing disorders [12,13]. The first case of disseminated $M$. abscessus subsp. bolletii infection in a young adult patient was described by Koh et al. [13]. Our patient was affected by ICL, a rare cause of immunodysfunction that, similar to HIV infection, causes a depletion of $\mathrm{CD} 4+\mathrm{T}$ lymphocytes. ICL diagnosis was confirmed by persistent $\mathrm{CD} 4+\mathrm{T}$ lymphocytes count under 300cells $/ \mu \mathrm{L}$ in the absence of HIV infection and common variable immunodeficiency.

The clinical spectrum of ICL ranges from an asymptomatic laboratory abnormality to life-threatening complications. ICL is typically revealed by the emergence of 
Table 1 Anti-microbial drug susceptibility test results for Mycobacterium abscessus subsp. bolletii isolate

\begin{tabular}{|c|c|c|}
\hline Anti-microbial agent & Minimum inhibitory concentration (MIC) $(\mu \mathrm{g} / \mathrm{mL})$ & Interpretive criteria [7] \\
\hline Amikacin $^{a}$ & 32 & Intermediate \\
\hline Amoxicillin clavulanate $^{b}$ & $>256$ & Resistant \\
\hline Cefotaxime $^{c}$ (disk, 30 $\left.\mu \mathrm{g}\right)$ & $>64$ & Resistant \\
\hline Cefoxitin $^{b}$ & $<8$ & Susceptible \\
\hline Ciprofloxacin ${ }^{a}$ & $>16$ & Resistant \\
\hline Clarithromycin $^{a}$ & 16 & Resistant \\
\hline Cotrimoxazole ${ }^{c}$ (disk, 1.25/23.75 $\mu \mathrm{g}$ ) & $>8$ & Resistant \\
\hline Erythromycin ${ }^{c}$ (disk, 15 $\left.\mu \mathrm{g}\right)$ & $>8$ & Resistant \\
\hline Imipenem $^{b}$ & 0.75 & Susceptible \\
\hline 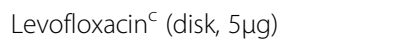 & $>4$ & Resistant \\
\hline 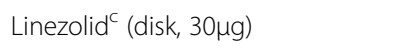 & $>8$ & Intermediate/Resistant \\
\hline Minocycline ${ }^{c}$ (disk, 30 $\left.\mu \mathrm{g}\right)$ & $<4$ & Susceptible \\
\hline 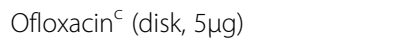 & $>8$ & Resistant \\
\hline Teicoplanin $^{b}$ & $>256$ & Resistant \\
\hline 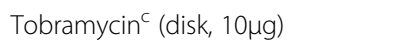 & $>8$ & Resistant \\
\hline Tigecycline $^{b}$ & $>256$ & Resistant \\
\hline Vancomycin $^{\mathrm{b}}$ & $>256$ & Resistant \\
\hline
\end{tabular}

${ }^{\mathrm{a}}$ Broth microdilution; ${ }^{\mathrm{b}}$ Etest ${ }^{\circledR}$ (AB Biodisk) at $30^{\circ} \mathrm{C}$ for 72 hours incubation; ${ }^{\mathrm{c}}$ disk diffusion.

opportunistic infections mainly nontuberculous mycobacteria and Cryptococcus spp. [14,15]. Disseminated mycobacterial infections have been rarely reported in patients affected by ICL [16-18]. This is the first report of $M$. abscessus subsp. bolletii infection in a patient affected by ICL. Our patient presented with a multifocal spread of $M$. abscessus subsp. bolletii infection consisting in pulmonary, CNS, genital, lymphonodal, subcutaneous involvement. Pulmonary disease is an uncommon but clinically relevant entity caused by RGM, most often by the $M$. abscessus group [19].

$\mathrm{CNS}$ is a rare localization of RGM infection. The most common cause is dissemination from pulmonary infection [1]. The clinical presentation was dominated by the sudden appearance of seizures due to a frontal lobe nodular lesion. A cerebral localization of M. abscessus subsp. bolletii is highly probable due to the concomitant presence of chronic lung infection in a patient who is immunocompromised and the presented MRI findings.

Orchiepididymitis, lymphadenitis and subcutaneous nodules could be interpreted as other localizations presumably due to hematogenous spread from $M$. abscessus subsp. bolletii lung infection. Ultrasonographic and/or microscopic pictures were suggestive for mycobacterial infection $[12,20,21]$.

As other RGM, M. abscessus subsp. bolletii is characterized by an inherent resistance to first-line antituberculous drugs. In addition, it has been described as a multi-drug resistant mycobacterium, notably to clarithromycin but with intermediate resistance to amikacin [4]. In contrast, in our patient's case the isolate of $M$. abscessus subsp. bolletii presented resistance to clarithromycin and was susceptible to cefoxitin, imipenem and minocycline (Table 1). It has been reported by Wallace et al. that the activity of tigecycline in vitro against several species of RGM was 11 times higher than other tetracyclines [21]. Conversely, the present M. abscessus subsp. bolletii isolate was resistant to tigecycline despite its susceptibility to minocycline. These differences could be due either to variability in antibiotic susceptibility within $M$. abscessus species or to the employ of different laboratory methods [22]. The therapeutic choice of azithromycin and minocycline was determined by our patient's poor compliance and by its ability to cross the hematoencephalic barrier.

The natural course of our patient's infection seems to be indolent even if the few descriptions of $M$. abscessus subsp. bolletii respiratory infection show high lethality [9]. However, the appropriateness of the therapeutic regimen could have also been negatively affected by some inconsistencies of the susceptibility testing approach.

The actual prognosis is difficult to establish because of his concomitant ICL, which continuously predisposes patients to various opportunistic infections.

\section{Conclusions}

Accurate identification of RGM species is very important with regard to their different drug susceptibility patterns, clinical significance and prognosis [19]. The emergence of new multi-drug resistant species is a threat in 
consideration of the increasing number of patients with immune system impairment of different origin.

\section{Consent}

Written informed consent was obtained from the patient for publication of this case report and any accompanying images. A copy of the written consent is available for review by the Editor-in-Chief of this journal.

\section{Competing interests}

The authors declare that they have no competing interests.

\section{Authors' contributions}

$L S, R R$ and $L T$ analyzed and interpreted the data from our patient regarding clinical features, laboratory findings and computed tomography images. CM, CB and CC performed the microbiological analysis of the kidney samples. CC was a major contributor in writing the manuscript. All authors participated in analysis and interpretation of the data, and read and approved the final manuscript.

Received: 12 December 2011 Accepted: 13 June 2012

Published: 4 September 2012

\section{References}

1. Talati NJ, Rouphael N, Kuppalli K, Franco-Paredes C: Spectrum of CNS disease caused by rapidly growing mycobacteria. Lancet Infect Dis 2008, 8:390-398.

2. Leao SC, Tortoli E, Euzéby JP, Garcia MJ: Proposal that Mycobacterium massiliense and Mycobacterium bolletii be united and reclassified as Mycobacterium abscessus subsp. Bolletii comb. nov., designation of Mycobacterium abscessus subsp. abscessus subsp. nov. and emended description of Mycobacterium abscessus. Int I Syst Evol Microbiol 2011, 61:2311-2313.

3. Leao SC, Tortoli E, Viana-Niero C, Ueki SYM, Lima KVB, Lopes ML, Yubero J, Menéndez MC, Garcia MJ: Characterization of mycobacteria from a major Brazilian outbreak suggests that revision of the taxonomic status of members of the Mycobacterium chelonae - Mycobacterium abscessus group is needed. J Clin Microbiol 2009, 47:2691-2698.

4. Adékambi T, Berger P, Raoult D, Drancourt M: rpoB gene sequence-based characterization of emerging non-tuberculous mycobacteria with descriptions of Mycobacterium bolletii sp. nov., Mycobacterium phocaicum sp. nov. and Mycobacterium aubagnense sp. nov. Int I Syst Evol Microbiol 2006, 56:133-143.

5. Adékambi T, Colson P, Drancourt M: rpoB-based identification of nonpigmented and late pigmenting rapidly growing mycobacteria. J Clin Microbiol 2003, 41:5699-5708.

6. NCCLS: Susceptibility testing of Mycobacteria, Nocardia, and Other Aerobic Actinomycetes. Approved Standard M24-A. Philadelphia: Wayne; 2003.

7. Brown-Elliot BA, Wallace RJ: Clinical and taxonomic status of pathogenic non pigmented or late-pigmenting rapidly growing mycobacteria. Clin Microbiol Rev 2002, 15:716-746.

8. Adékambi T, Drancourt M: Mycobacterium bolletii respiratory infections. Emerg Infect Dis 2009, 15:302-305.

9. Viana-Niero C, Lima KV, Lopes ML, Rabello MC, Marsola LR, Brilhante VC, Durham AM, Leão SC: Molecular characterization of Mycobacterium massiliense and Mycobacterium bolletii in isolates collected from outbreaks of infections after laparoscopic surgeries and cosmetic procedures. J Clin Microbiol 2008, 46:850-855.

10. Centers for Disease Control (CDC): Unexplained CD4+ T-lymphocyte depletion in persons without evident HIV infection. Morb Mortal Wkly Rep 1992, 41:541-545.

11. Roux AL, Catherinot E, Rippoll F, Soismier N, Guterriez C, Vincent V: Mycobacteries non-tuberculeuses et mucoviscidoses: enquête française de prevalence. Paris: Abstract 9ème Colloque des Jeunes Chercheurs en Mucoviscidose; 2008.

12. Griffith DE, Aksamit T, Brown-Elliott BA, Catanzaro A, Daley C, Gordin F, Holland SM, Horsburgh R, Huitt G, lademarco MF, Iseman M, Olivier K, Ruoss S, von Reyn CF, Wallace RJ Jr, Winthrop K: ATS Mycobacterial Diseases Subcommittee, American Thoracic Society; Infectious Disease Society of
America: An official ATS/IDSA statement: diagnosis, treatment, and prevention of nontuberculous mycobacterial diseases. Am J Respir Crit Care Med 2007, 175:367-416.

13. Kho WJ, Kwon OJ, Lee NY, Kook YH, Lee HK, Kim BJ: First case of disseminated Mycobacterium bolletii infection in a young adult patient. J Clin Microbiol 2008, 47:3361-3366.

14. Luo L, Li T: Idiopathic CD4 lymphocytopenia and opportunistic infection an update. FEMS Immunol Med Microbiol 2008, 54:283-289.

15. Zonios DI, Falloon J, Bennett JE, Shaw PA, Chaitt D, Baseler MW, Adelsberger JW, Metcalf JA, Polis MA, Kovacs SJ, Kovacs JA, Davey RT, Lane HC, Masur H, Sereti I: Idiopathic CD4+ lymphocytopenia: natural history and prognostic factors. Blood 2008, 112:287-294.

16. Vargas J, Gamboa C, Negrin D, Correa M, Sandoval C, Aguiar A, Prieto M, Rodriguez-Morales AJ, De Waard J, Yakrus M: Disseminated Mycobacterium mucogenicum infection in a patient with idiopathic CD4+ T lymphocytopenia manifesting as fever of unknown origin. CID 2005, 41:759-760.

17. Nigg AP, Sternfeld T, Gamarra F, Krötz M, Belohradsky BH, Bogner JR: Recurring disseminated Mycobacterium avium infections in an HIVnegative patient [in German]. Dtsch Med Wochenschr 2005, 130:1369-1372.

18. Muttarak M, Peh WC: Case 91: tuberculous epididymo-orchitis. Radiology 2006, 238:748-751.

19. Piersimoni C, Scarparo C: Pulmonary infections associated with nontuberculous mycobacteria in immunocompetent patients. Lancet Infect Dis 2008, 8:323-334.

20. Morales P, Ros JA, Blanes M, Pérez-Enguix D, Saiz V, Santos M: Successful recovery after disseminated infection due to Mycobacterium abscessus in a lung transplant patient: subcutaneous nodule as first manifestation - a case report. Transplant Proc 2007, 39:2413-2415.

21. Wallace RJ Jr, Brown-Elliott BA, Crist CJ, Mann L, Wilson RW: Comparison of the in vitro activity of the glycylcycline tigecycline (formerly GAR-936) with those of tetracycline, minocycline, and doxycycline against isolates of nontuberculous mycobacteria. Antimicrob Agents Chemother 2002, 46:3164-3167

22. Martín-de-Hijas NZ, Fernández-Roblas R, Fernandez-Martínez Al, Gadea I, Esteban J: Usefulness of the Etest for studying tigecycline susceptibility of non-pigmented rapidly growing mycobacteria. Int J Antimicrob Agents 2008, 32:366-367.

doi:10.1186/1752-1947-6-277

Cite this article as: Colomba et al:: Probable disseminated Mycobacterium abscessus subspecies bolletii infection in a patient with idiopathic CD4+ T lymphocytopenia: a case report. Journal of Medical Case Reports 2012 6:277.

\section{Submit your next manuscript to BioMed Central and take full advantage of:}

- Convenient online submission

- Thorough peer review

- No space constraints or color figure charges

- Immediate publication on acceptance

- Inclusion in PubMed, CAS, Scopus and Google Scholar

- Research which is freely available for redistribution 